\title{
Current World Health Organization-sponsored studies in the chemotherapy of leprosy
}

\author{
D. DAUMERIE \\ World Health Organization, Geneva, Switzerland
}

Until the early 1980s, dapsone monotherapy administered to known patients was used for control of leprosy. Dapsone was usually administered in a dosage of $100 \mathrm{mg}$ daily for a minimum of 5 years to patients with paucibacillary (PB) leprosy, and for life to patients with multibacillary (MB) leprosy. Because it was a weakly bactericidal and slowly acting drug, dapsone monotherapy led to poor patient compliance and the emergence of dapsone-resistant strains of Mycobacterium leprae.

Since 1982, the multidrug therapy (MDT) regimens recommended by the World Health Organization (WHO), which include a combination of rifampicin, clofazimine and dapsone for MB leprosy and rifampicin plus dapsone for PB leprosy, have been applied in national leprosy control programmes in all of the leprosy endemic countries in the world. This therapy has proved to be a most reliable and practical method of treating leprosy.

The introduction of MDT in 1982 has resulted in a dramatic reduction of the prevalence of leprosy, from 5.4 million registered cases in 1985 to $0 \cdot 8$ million in 1999. By the end of 1999 , more than 10 million patients had been cured by MDT. Follow-up of the large number of patients cured by MDT has revealed very low relapse rates after stopping treatment; the cumulative risk of relapse is less than $1 \%$ over a period of 9 years for both $\mathrm{MB}$ and $\mathrm{PB}$ leprosy.

Despite the success of the standard WHO MDT regimens, there is a need for more effective combined drug regimens. Several WHO-sponsored programmes of research in the chemotherapy of leprosy are currently in progress. The purpose of this report is briefly to describe the programmes and their current status.

\section{The THEMYC-sponsored ofloxacin multicentre trial}

Since the late 1980s, three additional bactericidal antileprosy drugs have been available: ofloxacin, a fluoroquinolone; clarithromycin, a macrolide; and minocycline, a tetracycline. All three act by different mechanisms. These drugs offer the potential of increased effectiveness and shortened duration of antileprosy chemotherapy. In addition, they may prove useful against strains of $M$. leprae that are resistant to the drugs currently in use, especially those resistant to rifampicin.

Therefore, in 1991 and 1992, the WHO launched a large scale multicentre field trial, the main objectives of which are to evaluate the efficacy, acceptability and feasibility of ofloxacin-containing combined regimens in a randomized, double-blind, controlled clinical trial among both MB and PB leprosy patients. One of the regimens in the trial is a combination of rifampicin plus ofloxacin daily for 4 weeks for both MB and PB leprosy. 
The other two regimens, both for MB leprosy, are the WHO-recommended MDT for 1 year, with or without supplementation by daily ofloxacin during the first 4 weeks. The control regimen is the standard WHO-recommended MDT regimen for 24 months.

Thus, the current trial has six arms, four for MB and two for PB leprosy. For MB leprosy, the four arms are: (i) WHO MDT for 2 years; (ii) WHO MDT for 1 year, (iii) WHO MDT for 1 year supplemented by ofloxacin daily for the first 4 weeks; (iv) ofloxacin plus rifampicin for 4 weeks. For PB leprosy, the two arms are: (i) WHO MDT for 6 months; and (ii) ofloxacin plus rifampicin for 4 weeks. Fifteen centres from eight endemic countries are participating in this trial. The intake of nearly 4000 patients was completed in June 1994, and treatment was completed in December 1996. Follow-up will continue until December 2003, and final results are expected to be available by mid-2004.

\section{The LEP-sponsored single-dose ROM trial for PB leprosy}

The objective of this study is to evaluate the therapeutic efficacy of rifampicin combined with ofloxacin plus minocycline (ROM), administered as a single dose, for the treatment of all skin smear-negative PB cases. The study being carried out in India is designed as a double-blind, randomized, controlled trial, with two arms. ROM is administered as a single dose to one group of patients, who are administered a placebo in place of the standard PB MDT for the remaining 6 months. The other group receives the standard PB MDT for 6 months, together with a placebo in place of the dose of ROM on day 1. Efficacy will be measured in terms of clinical response and relapse. The total duration of the study will be 54 months: 12 months for intake, 6 months for treatment, and 36 months of post-treatment follow-up.

The intake of patients began in April 1998, and was completed in June 1999. The first patients recruited into this trial began treatment in April 1998, and treatment of the last patients was completed in March 2000. The phase of follow-up began in December 1998, and will be completed in March 2003. Final results should be available by the end of 2003 .

\section{LEP-sponsored trial of monthly administration of ROM for MB and PB leprosy}

The main objective of this trial, which is being carried out in Myanmar, Guinea and Senegal, is to study in both MB and $\mathrm{PB}$ leprosy patients the ef ficacy of once-monthly doses of ROM in terms of treatment failure or relapse, in order to assess the robustness of the study regimens. MB patients were administered a single, supervised monthly dose of ROM for 12 months, whereas PB patients received a single, supervised monthly dose of ROM for 3 or 6 months.

Patient intake was begun in 1995 and 1996, and was completed by June 1997. Treatment of the first patients began in March 1995, and treatment of the last patients was completed in December 1999. Follow-up, which is planned for 5-7 years after completion of treatment, is to be completed in 2006, and the final results should become available in mid- 2007 .

\section{THEMYC-sponsored experimental chemotherapy}

Recently, three drugs, rifapentine (a rifamycin), moxifloxacin (a fluoroquinolone), and HMR (a macrolide), have been reported to show impressive bactericidal activity against $M$. leprae 
in the mouse footpad system, and a single dose of a combination of rifapentine, moxifloxacin and minocycline (PMM) was found to be more bactericidal than was rifampicin alone, as reported by Professor Baohong Ji in this Workshop.

\section{DISCUSSION}

Professor Grosset: I agree with Dr Daumerie that efforts to control both leprosy and tuberculosis suffer from operational problems. The treatment of tuberculosis has been standardized for the last 40 years. However, its implementation in the field is very difficult. There is much the same problem for leprosy.

Drvan Brakel: I believe that a meeting similar to this must be held to consider operational issues, which are at least as important as the scientific issues under discussion in this Workshop.

Professor Ji: One may approach operational problems both operationally and scientifically. In some African countries, which I have visited during the past few years, it is the practice, immediately after diagnosis, to issue to the patient his entire supply of medication, enough for 6 months for PB patients, and enough medication for 12 months for MB patients. It is this that stimulated me to think about the problems of rifampicin resistance and of a fully supervisable regimen. The solutions to these problems should provide solutions to some important operational problems.

Dr Daumerie: Your statement is very important. I have always believed that researchers should meet with control personnel before setting their priorities. 